\title{
Creating Adventurous and Mindful Physicians: Expanding Wilderness Orientation Programs to Medical School
}

Alec Bernard, BS; Jacquelyn Kercheval, BS; Alexander D Blaty, MD; Heather Burrows, MD PhD

\section{ABSTRACT}

\section{Background}

Wilderness orientation programs are used by several undergraduate institutions to assist students in their transition from high school to college. These programs have demonstrated efficacy in improving educational readiness and building resilience. Although a few similar programs have been offered to incoming medical students, no data currently exists showing program impact on participating students. Such programs could be especially useful for medical students because of their potential ability to mitigate medical school burnout. In the summer of 2017, a pilot wilderness preorientation program, CAMP (Creating Adventurous and Mindful Physicians), was developed at the University of Michigan Medical School. The program included outdoor skill building and activities around mindfulness and value articulation.

\section{Program Overview}

Fifteen students were recruited for a three-day, two-night backpacking trip, which took place immediately before the start of classes. The trip's curriculum was developed from established undergraduate programs and tailored to the unique requirements of a medical school program.

\section{Outcomes Assessment}

One goal of the pilot program was to gauge student perceptions of the trip's efficacy to better inform the expansion of CAMP to more students. To assess this, a basic survey was developed based on the Maslach burnout index with some additional shortanswer entries. Many participants acknowledged an improvement in various measures associated with burnout, including emotional strength and energy. Students cited factors that contributed to 
the positive outcomes of building a culture of support, bonding, and readiness for medical school. Some factors mentioned included the wilderness setting outside of academics, the opportunity to overcome challenges, the activities focused on reflection, and the ability to bond with classmates.

\section{Conclusions}

Results from the 2017 pilot program suggest that the trip facilitated a sense of community and preparedness among trip participants and positively impacted the adjustment to medical school. CAMP at the University of Michigan Medical School is one of the first wilderness orientation programs designed specifically for medical students. Based on the apparent success of this pilot program and the potential to create a new body of research, UMMS has agreed to formally fund the expansion of the program for the summer of 2018 to include up to 60 incoming students on multiple trips.

\section{Introduction}

$\mathbf{O}$ ver the last 50 years, wilderness orientation programs have become commonplace to help students transition from high school to college. The goals of these programs are varied, but they typically include reducing attrition rates, increasing academic performance, improving social and emotional development, increasing resilience, and expanding positive attitudes toward the institution. Studies examining the outcomes of these programs in undergraduate institutions have largely supported their positive impact and role in preparing students for college. ${ }^{1}$ The positive effects of these programs also last well beyond the students' undergraduate years. ${ }^{2}$

The transition to medical school is similar to the transition to the undergraduate years: students are often in a new physical location with a limited support system, uncertainty runs high about how the next few years will unfold, and anxiety about the prospect of burn out is pervasive. For these reasons, adding wilderness orientation programs to medical school seems like a natural pairing. However, only a few such programs currently exist, and no research has yet been conducted on their effectiveness.

To address this gap, the University of Michigan Medical School (UMMS) piloted a wilderness orientation program in the summer of 2017. The high-level goal of the program was to preemptively mitigate burnout by establishing a supportive community and train incoming medical students on wellness and mindfulness techniques. In this article, we describe the process of adapting existing undergraduate programs to a medical school context. We also assess outcomes of the pilot trip to determine if it successfully built a community and established a foundation for wellness among the participants.

\section{Literature Review}

Current undergraduate outdoor orientation programs vary in design from weekend retreats to wilderness expeditions lasting over a week. ${ }^{3}$ These programs often involve team-building, adventure, and wilderness activities, as well as discussions about the transition to college. They include teaching in a variety of domains, including hard skills, soft skills, and university-specific competencies. The hard skills comprise technical skills necessary for specific outdoor tasks, including everything from stove operation to map reading to canoe-paddling techniques. In contrast, soft skills cover leadership development and learning about group dynamics. ${ }^{4}$ Interpersonal success has been identified as an important factor in student retention, and outdoor orientation programs provide the opportunity to strengthen communication and social skills to improve retention and academic success. ${ }^{5}$

Outdoor orientation programs have also been shown to positively impact incoming students' sense of place and community. ${ }^{6}$ Students in successful orientation programs have self-identified growth in the areas of social competence, achievement motivation, 
intellectual flexibility, task leadership, emotional control, active initiative, and self-confidence. ${ }^{4}$ These positive changes were attributed to four factors of the programs: a wilderness setting, small group size, fellow participants, and peer leadership. ${ }^{3}$ Wilderness orientation programs promote these four factors by ensuring social integration, physical activity, significant positive interactions with faculty, and preparation for university life. ${ }^{7}$

While the success of outdoor orientation programs in an undergraduate setting has been well-supported, their use in medical school is just beginning to be explored. Limited peer-reviewed literature is available on wilderness orientation programs in medical school, but programs are starting to appear at a select few schools across the country. Stanford Medical School's SWEAT (Stanford Wilderness Education Active Orientation Trip) is an orientation program with a participation rate of around $90 \%$ of the incoming class. These trips last 4 days and 3 nights and have difficulty levels ranging from car camping to backpacking with long hikes. Columbia University College of Physicians and Surgeons leads a trip called P-SOOP, which has 32 participants, 10 second- and fourth-year leaders, and 3 support staff. It is a 3-day, 2-night backpacking trip in the Catskill mountains, designed to allow students to get to know each other in a unique environment and ease the transition to medical school. A third program, Pre-Op at UC San Diego Medical School, lasts 2 nights and has the express goal of helping students get to know the new community while having fun in the outdoors. The trip includes challenges, camping, and bonding exercises and is run by UCSD's Recreation-Outback Adventures program along with second-year medical students. Although these programs exist and other medical schools likely have dedicated orientation programs, there is a current lack of literature assessing the development and impact of these programs on medical student well-being.

Burnout is one critical aspect of medical student well-being that has received recent interest in the literature. Although the research on physician burnout extends back decades, the importance of considering burnout as early as medical school is still a fairly novel idea. The literature is rife with studies discussing the prevalence and impact of physician burnout, which has affected over 50\% of practicing physicians..$^{8,9}$ Its presence in the literature has been substantial for some time because studies have found that physician burnout trickles down to affect patient well-being as well. ${ }^{9}$ Although burnout has historically been studied on the physician level, more recent research suggests that burnout is beginning as early as the medical school years, with one meta-analysis estimating its prevalence at 45\%-71\% among US medical students. ${ }^{10}$ Burnout is often classified as a three-pronged state of being, comprising emotional exhaustion, depersonalization, and a low sense of personal accomplishment. Students' struggles with burnout manifest across all three of these components, with one study reporting that 35\%,26\%, and 31\% of students have experienced high emotional exhaustion, high depersonalization, and a low sense of personal accomplishment, respectively. ${ }^{11}$

To mitigate the harmful effects of burnout, a variety of interventions have been proposed and studied at the physician level. West et al (2016) conducted a meta-analysis of the literature addressing physician burnout and found that existing interventions are capable of significantly reducing burnout prevalence. Interventions at both the individual and organizational levels appear to help, with individuallevel strategies focusing on facilitating small-group curricula; training on stress management, self-care, and communication skills; and addressing the concept of "belonging" among physicians. ${ }^{12}$

While physician-level findings are likely transferable to medical students, studies have begun to propose interventions specifically to address burnout during medical school. Broadly, many of the recommendations look at "generating wellness during medical training,"10 and there is an understanding that the structure of medical school offers an opportunity to integrate a focus on wellness into this critical training period. ${ }^{13}$ One study conceptualizes medical students' 
ability to combat burnout as a reservoir, with various factors simultaneously filling and depleting that reservoir. Interventions that bolster psychosocial support, social activities, mentorship, and intellectual stimulation are believed to fill the reservoir, thereby combating burnout and bolstering resilience. ${ }^{13}$

Orientation trips, which provide social support, training on wellbeing, physical and intellectual stimulation, and more, are one possible medium through which burnout can be combated early in a medical student's career. However, no research has been yet conducted to address the possible connection.

\section{CAMP at UMMS}

This past year a group of incoming, first-year students at UMMS developed a wilderness orientation program that mirrored the successes of similar programs in undergraduate settings. To design the trip's curriculum, the students studied and adapted curricula from undergraduate university programs to transfer the benefits and tailor the content to the unique challenges of medical school. The finalized program curriculum, which ended up with the name CAMP (Creating Adventurous and Mindful Physicians), included team-building activities, meaning-in-medicine exercises, mindfulness training, backpacking lessons, and wilderness medicine instruction. The trip leader was an experienced outdoor educator and incoming medical student who drew from his past experiences and also incorporated new information designed to meet the demands of a medical school-specific trip. The program was designed to increase students' feelings of belonging and readiness for medical school and improve their emotional states. Longer term, it was designed to provide tools for resilience and instill a culture of support within the starting class.

The inaugural, three-day, two-night backpacking trip was held in the Pinckney State Recreation Area during the week before the UMMS White Coat Ceremony in July 2017. CAMP began with a meeting and discussion the night before the backpack adventure to develop a shared contract of values based on the particular goals and needs of the group. Per this "Full-Value Contract," each student agreed to fully value each other, the group, and the experience as a whole. During the hike, camping- and trail-specific skills were taught, with some of the more experienced students having opportunities to lead in the areas where they felt comfortable. During breaks and lunches, discussions were facilitated around the demands of medical school and comparisons were drawn between experiences on the trip and participants' future as medical students. Mindfulness meditation was incorporated in small sessions throughout the day, and students completed a writing exercise in which each composed a letter to their future selves. Their letters were mailed to them six months after the start of medical school.

On the final night of the trip, campers were joined by five fourth-year medical students and the Assistant Dean for Curriculum. The purpose of this segment of the trip was to promote interclass and student-faculty bonding and alleviate some of the apprehension about starting medical school. In addition to presenting several short wilderness medicine topics to students, upperclassmen joined the incoming medical students in a "Fears in a Hat" exercise, in which students were provided with an outlet to discuss their concerns about medical school with each other and the upperclassmen.

CAMP participants were recruited with a post on the "2017 UMMS Admitted Students" Facebook group page, and a survey was initially sent out to determine interest. 32 students filled out the initial survey indicating an interest in attending, with 15 students eventually participating. The students who did not attend listed reasons such as attending a different medical school or not being able to schedule apartment move-in before the time of the trip. Participants were self-selected and included four males and 11 females, including the trip leader. No experience was necessary, and the students' camping experience ranged from none to experienced wilderness guides. While specific demographic data are not available, the participants represented a mix of students, from 
those who began medical school immediately upon college graduation to those who had taken one or more years off before starting medical school. The age range was 21 to 27.

\section{Assessment of the Trip}

Because this trip was designed by a single student as a pilot program to test the feasibility of developing a wilderness orientation program, the focus was on establishing the curriculum and coordinating logistics more than designing a robust, quantitative assessment of its efficacy. Future iterations of this wilderness trip will include more thorough quantitative analyses to assess the significance of the impact of this program on incoming students' well-being. However, a basic survey (Appendix A [online only]) was established prior to the trip to gauge whether the outing impacted some of the more common indicators of burnout, including students' emotional state, energy, and senses of belonging and preparedness leading up to medical school.

The same survey was sent twice to all 15 participants (once prior to and once following the trip). Because the survey's purpose was to broadly assess burnout-related sentiment pre- and posttrip, questions were largely based on the Maslach
Burnout Inventory, one of the most well-studied and verified tools for measuring burnout risk. ${ }^{7}$ Participants were also given text-entry space to offer any comments about the trip at large.

Because of the basic nature of the survey and an incomplete response rate (12 of the 15 students responded to the majority of questions before and after the trip), statistical analysis of the results was not performed. A high-level set of results is presented in Table 1 to provide readers with a sense of this trip's efficacy and the potential impact similar trips can have in the future.

When the pre- and post-trip survey responses are compared individually, results suggest that many participants saw an improvement in various measures associated with burnout (Table 1). For all but one respondent, emotional fortitude and energy were either reinforced or improved following the trip. These two characteristics are strongly associated with burnout, and when students were explicitly asked about their fear of burnout, the pre- and post-trip comparison results were similar: all but one student felt similarly or less worried about burnout following the trip. Results also suggest that the trip helped to facilitate a sense of community (i.e., belonging) and preparedness among trip participants.

TABLE 1: Changes in individual responses between pre- and post-trip Emotional Fortitude Energy Sense of Belonging Fear of Burnout Preparedness

\begin{tabular}{llllll} 
Improvement & $7(58.3 \%)$ & $6(50.0 \%)$ & $4(33.3 \%)$ & $4(33.3 \%)$ & $6(50.0 \%)$ \\
\hline Same & $4(33.3 \%)$ & $5(41.7 \%)$ & $8(66.7 \%)$ & $7(58.3 \%)$ & $5(41.7 \%)$ \\
\hline Decrease & $1(8.3 \%)$ & $1(8.3 \%)$ & $0(0.0 \%)$ & $1(8.3 \%)$ & $1(8.3 \%)$
\end{tabular}

Notes:

(1) The data presented here were collected based on responses to the following five survey measures:

Emotional fortitude: "I feel emotionally drained."

Energy: "l feel energetic."

Sense of belonging: "I belong at Michigan Medical School."

Fear of burnout: "I think I will get burned out during medical school."

Preparedness: "I feel mentally prepared for medical school."

(2) Survey responses were scored on a Likert Scale ranging from 1 to 5. The scale assessed the respondents' range of disagreement through agreement for each measure. 
It is important to note the limitations of these results. The purpose of the initial survey was not to quantitatively inform the body of literature on the nature of these types of trips, but rather to help the students in charge of the trip better plan for subsequent trips and generate more robust measurement tools in the future. Further, the small sample size prohibits statistical analyses of the data, as does the fact that the study examined only one program at UMMS and did not include a control group. Of note, the survey was looking at proxy measures of burnout, which may not appropriately assess all potential benefits of the trip. Additionally, while participating students represented a wide variety of backgrounds and experience levels, students in the program were self-selected and may represent different characteristics than those who chose not to participate.

The survey provided free-text space for participants to comment on the trip at large, and these comments were analyzed to identify themes across participants. The post-trip comments from the various participants shared a number of themes, including connection with other students, a desire for the trip to continue in future years, increased comfort with starting medical school, and a sense of bonding and community. Community-building was especially emphasized in participants' responses, with frequent remarks about the quality of the friendships that were made and a sincere sense of feeling welcomed into the medical school community. Additionally, students mentioned the diversity of experience levels and conversation topics as aspects they enjoyed about the trip. Finally, although "burnout" was not a term explicitly used by participants, their responses alluded to a sense of increased capacity to deal with future adversity. Students specifically stated that they felt much more mentally and emotionally prepared to begin medical school following the trip.

Some of the factors that students mentioned as contributing to the positive outcomes of building a culture of support, bonding, and readiness for medical school included the wilderness setting (outside of academics), the opportunity to overcome challenges, the reflection activities, and the chance to bond with classmates.

The complete set of participant responses can be found in Appendix B (online only). Future iterations of this wilderness trip will include more thorough quantitative tools to assess the impact of this program on incoming students' well-being.

\section{Discussion}

Although this was a pilot wilderness orientation program and the number of participants was small, an initial analysis of the data and qualitative survey information suggests that the program had a positive impact on participants. Its apparent success is likely attributable to two overall factors: first, the curriculum design was informed by past successes from undergraduate preorientation programs, and second, medical students' susceptibility to burnout makes this population more likely to benefit from a well-structured program. In terms of curriculum, CAMP was held by design in a wilderness area that included surmountable backpacking challenges; provided a guided space for participants to connect; and was led by a peer with help from older students. Additionally, a dean from the medical school came to meet the trip attendees for dinner on the last night and participated in related bonding activities. These aspects align closely with the factors identified as most impactful in various undergraduate programs, and survey responses suggest the program design likely had its desired positive impact.

The findings will be especially useful in informing the design of future wilderness orientation trips at UMMS. Because of the positive feedback from participants in this pilot program, a team of medical students is currently working to expand CAMP and offer it to a greater number of incoming students in the summer of 2018. As part of that trip, a more robust survey is being designed to enable an examination of longitudinal benefits in comparison with a control group. This control group could be 
made up of students who expressed interest in attending but were unable to because of scheduling constraints. Comparing rates of dropout, scores on school-wide surveys of well-being, and academic measurements between these groups would provide a deeper look into the benefits of a wilderness orientation program and its effect on preventing medical school burnout. Additional data could be collected from similar programs at other medical schools, such as Stanford's SWEAT program.

\section{Conclusion}

The CAMP program at the University of Michigan Medical School is one of a handful of wilderness orientation programs tailored specifically to medical students. In its pilot summer of 2017, a comparison of pre- and post-trip survey results suggests that the novel curriculum successfully facilitated an increase in students' emotional fortitude, sense of belonging, and excitement about medical school. These findings are very promising given the current medical student burnout literature, which does not assess the ability of orientation programs to preemptively mitigate burnout. Based on the apparent success of this pilot program, as well as the potential to create a new body of research, UMMS has agreed to formally fund the expansion of the program for the summer of 2018 to include up to 60 incoming students. A more rigorous case-control survey design will enable a more thorough statistical analysis of the expanded version of CAMP with the goal of better exploring the potential relationship between orientation programs and burnout among medical students.

\section{Acknowledgements:}

We would like to acknowledge the University of Michigan Medical School Admissions team for funding CAMP, Dean Michelle Daniel for her participation and support, and the Wilderness Medicine Interest Group for their instruction of a wilderness medicine skill lesson.

\section{Citations:}

1. Bell BJ. Wilderness orientation: exploring the relationship between college preorientation programs and social support. J Exper Ed. 2006;29(2):145-167.

2. Gass MA, Garvey DE, Sugerman DA. The long-term effects of a first-year student wilderness orientation program. J Exper Ed. 2003;26(1):34-40.

3. Bell BJ, Holmes MR, Williams BG (2010). A census of outdoor orientation programs at four-year colleges in the United States. J Exper Ed, 2010;33(1):1-18.

4. Frauman E, Waryold D. Impact of a wilderness orientation program on college student's life effectiveness. JOREL, 2009;1(2):189-207.

5. McCroskey JC, Booth-Butterfield S, Payne SK The impact of communication apprehension on college student retention and success. Comm Quarterly. 1989;37(2):100-107.

6. Austin ML, Martin B, Yoshino A, Schanning K, Ogle, $\mathrm{DH}$, \& Mittelstaedt R. The intersection of community and place in an outdoor orientation program. JOREL, 2010;2(1):74-92.

7. Wolfe BD, Kay G. Perceived impact of an outdoor orientation program for first-year university students. J Exper Ed. 2011;34(1):19-34.

8. Gunderson L. Physician burnout. Ann Intern Med, 2001;135(2):145-148.

9. Epstein R M, Privitera, MR. Doing something about physician burnout. Lancet, 2016;388(10057):2216-2217.

10. Ishak W, Nikravesh R, Lederer S, Perry R, Ogunyemi D, Bernstein C. Burnout in medical students: a systematic review. Clin Teach, 2013;10(4):242-245.

11. Dyrbye LN, Thomas MR, Huntington JL, et al. Personal life events and medical student burnout: a multicenter study. Acad Med, 2006;81(4):374-384.

12. Bell B J, Gass M, Vlamis E. Effects of college adventure orientation programs on student development behavior. J ExperEd. 2011;34(2):127-148.

13. Dunn LB, Iglewicz A, Moutier C. A conceptual model of medical student well-being: promoting resilience and preventing burnout. Acad Psychiatry. 2008;32:44-53. 